\title{
La salida de campo como recurso didáctico para enseñar ciencias. Una revisión sistemática
}

\author{
David Aguilera ${ }^{1, a}$ \\ ${ }^{1}$ Departamento de Didáctica de las Ciencias Experimentales, Universidad de Granada. Granada. España \\ adavidaguilera@ugr.es
}

[Recibido: 2 enero 2018. Revisado: 10 Abril 2018. Aceptado: 6 Junio 2018]

\begin{abstract}
Resumen: El estudio de revisión aquí presentado tiene como propósitos: (1) aportar una panorámica del desarrollo de la línea de investigación que se ocupa del estudio de las salidas de campo como recurso didáctico en Didáctica de las Ciencias Experimentales; y, (2) sintetizar las principales implicaciones educativas referidas a la misma. Así, se revisaron los artículos publicados entre el año 2000 y 2017 y alojados en las bases de datos de Web of Science y Scopus. Los resultados obtenidos giran en torno a los campos: (1) autor/es y año; (2) país; (3) disciplina científica; (4) etapa educativa; (5) contexto; (6) metodología; y (7) implicaciones educativas. Los resultados principales apuntan a la irrupción de la producción científica sobre esta línea de investigación, a pesar de no abundar autores muy prolíficos en ella. Además, se ha comprobado que Estados Unidos es el país donde se realizan mayor número de trabajos, mientras que la etapa educativa preferente para ejecutar las salidas de campo es la Educación Primaria. Los espacios naturales se colocan como el contexto más visitado y el Medio Ambiente, la Biología y la Geología como las disciplinas predilectas para realizar salidas de campo. Finalmente, las principales implicaciones educativas señalan la promoción de actitudes y emociones positivas, así como la adquisición de los contenidos como puntos fuertes de las salidas de campo.
\end{abstract}

Palabras clave: Enseñanza de las ciencias; salidas de campo; revisión sistemática; bibliográfico.

Field trip as a didactic resource to teach sciences. A systematic review

Abstract: This review study aims to: (1) provide an overview of development of the educational research about the use of field trips as a teaching resource in Didactics of Experimental Sciences; and (2) synthesize the main educational implications referred to it. Thus, the articles published between 2000-2017 and hosted in Web of Science and Scopus databases were reviewed. The results approved around the fields: (1) author/s and year; (2) country; (3) scientific discipline; (4) educational stage; (5) context; (6) methodology; and (7) educational implications. The main results point to irruption of the scientific production on this line of investigation, in spite of not abounding prolific authors in her. In addition, we have proven that United States is the country where the greatest numbers of jobs have been carried out, while the preferential educational stage to carry out field trips is Primary Education. The natural spaces are placed as the most visited context and the Environment, Biology and Geology as the preferred disciplines for field trips. Finally, the main educational implications point to the promotion of positive attitudes and emotions, as well as the acquisition of contents as strengths of field trips.

Keywords: Science education; field trip; systematic review; bibliographic.

Para citar este artículo: Aguilera D. (2018) La salida de campo como recurso didáctico para enseñar ciencias. Una revisión sistemática. Revista Eureka sobre Enseñanza y Divulgación de las Ciencias 15(3), 3103. doi: 10.25267/Rev_Eureka_ensen_divulg_cienc.2018.v15.i3.3103

\section{Introducción}

Emplear la salida de campo, de ahora en adelante SC, como un recurso didáctico está lejos de ser algo novedoso en la enseñanza actual. Así, la corriente renovadora de la Escuela Nueva surgida a finales del siglo XIX y desarrollada en el siglo XX, donde encontramos a autores como John Dewey, Maria Montessori o Célestin Freinet, quienes promovieron la necesidad de salir fuera del aula como una parte fundamental en el proceso de enseñanza-aprendizaje del alumnado. Tan es así que Sorrentino y Bell (1970) establecen cinco propósitos para esta herramienta didáctica: 
(1) Propiciar experiencia.

(2) Estimular el interés y la motivación del alumnado hacia las ciencias.

(3) Atribuir relevancia al aprendizaje de las ciencias.

(4) Desarrollar las habilidades de observación y percepción.

(5) Favorecer el desarrollo personal y social.

No obstante, la frecuencia con la que se realizan SC, tradicionalmente en las áreas curriculares de Ciencias Experimentales y Sociales, es bastante baja (Pedrinaci, 2012). Ello provoca que la sensación de novedad en el alumnado o la mal entendida percepción de innovación educativa en el profesorado perduren en el tiempo durante la planificación y puesta en práctica de una SC en el ámbito escolar. Las razones contribuyentes a la situación descrita, habitualmente, están relacionadas con el número de alumnos por aula, el esfuerzo extra que debe realizar el profesorado, aspectos económicos y burocráticos, la escasez de materiales didácticos sobre estas actividades y la responsabilidad civil (Rebelo, Marques y Costa 2011).

Ante las situaciones expuestas, parece pertinente estudiar la producción científica que versa sobre la temática abordada como ya hicieron Mason (1980), quien encontró 43 trabajos referidos a las SC entre 1920 y 1980, Guisasola y Morentín (2007), quienes centraron su atención en las visitas escolares a los museos de ciencias y Amórtegui, Gavidia y Mayoral (2016), quienes analizaron trabajos que abordaban las salidas de campo para enseñar Biología, DeWitt y Storksdieck (2008) y Behrendt y Franklin (2014), quienes valoraron el potencial pedagógico de las SC. Asimismo, este estudio tiene entre sus propósitos resaltar el valor educativo de las SC en el área de Didáctica de las Ciencias Experimentales (DCE), así como mostrar el desarrollo de esta línea de investigación en lo que va de siglo XXI (2000-2017). La consecución de estos propósitos está vinculada con las siguientes preguntas de investigación:

(1) ¿Cuál ha sido la evolución de la producción científica en lo que va de siglo XXI?

(2) ¿Cuáles son los autores más prolíficos en esta línea de investigación? ¿Qué grado de colaboración existe entre los autores? ¿Se identifica algún grupo de investigación?

(3) ¿En qué países, etapas educativas, disciplinas científicas y contextos se desarrollan las investigaciones?

(4) ¿Qué metodologías de investigación se emplean para tal fin?

(5) ¿Qué implicaciones educativas aportan los estudios sobre las SC?

\section{La salida de campo: conceptualización y puesta en marcha}

Tal y como se apuntaba al inicio del manuscrito, la SC es un recurso didáctico cuyo uso se remonta a cientos de años. Una de las definiciones más empleadas es la propuesta por Krepel y Durrall (1981), quienes definen salida de campo como aquel viaje que realiza una escuela o una clase con una intención educativa, donde el alumnado puede interactuar con el entorno, experimentar y observar para asociar sus ideas con conceptos científicos a través de la experiencia. En este mismo sentido, Tal y Morag (2009) describen a las SC como aquellas actividades con fines educativos desarrolladas fuera del aula, en un ambiente interactivo, capaz de proveer al alumnado de experiencias. Más recientemente, Álvarez-Piñeros, Vásquez-Ortiz y Rodríguez-Pizzinato (2016) caracterizan la SC como la oportunidad de explorar, descubrir y redescubrir una realidad cercana o lejana para el alumnado, tratándose de un proceso donde el nombre de las «cosas» juega un papel esencial para poder observarlas, describirlas y explicarlas in situ, convirtiéndolas en objeto de investigación.

$\mathrm{Si}$ analizamos las definiciones expuestas, se encuentran tres coincidencias clave: es una actividad que tiene lugar fuera del aula, tiene un fin educativo y genera experiencia en el alumnado. Todo ello se presenta como una oportunidad para la consecución de algunos objetivos de la enseñanza de las ciencias, puesto que las SC: 
- Generalmente, tienen lugar en sitios atractivos para el alumnado (Orion 2001).

- Proporcionan una experiencia directa con aquello que se estudia en el aula, promoviendo la curiosidad del alumnado gracias a la actitud investigativa adoptada (Allen 2004).

- Inciden en el desarrollo social y personal del alumno (Gair 1997).

- Establecen conexiones entre la Ciencia, la Tecnología y la Sociedad (CTS), permitiendo el desarrollo de una ciudadanía más activa (Pedretti 2003).

En cuanto a la puesta en marcha de una SC, ésta se puede llevar a cabo de distintas formas, cada una con sus fortalezas y debilidades y con mayor o menor éxito. Uno de los modelos referidos al diseño y ejecución de SC más citados es el descrito por Orion (2007), el cual consta de tres fases:

(1) La primera fase denominada «construcción de significados» puede tener una duración variada, dependiendo de los objetivos de la SC. Su propósito es preparar al alumnado para la actividad, tratando de reducir el factor «novedad» que surge en el alumnado (Orion y Hofstein 1994). Dicho factor se compone de tres aspectos: cognitivo, geográfico y psicológico. Así, la novedad cognitiva responde a los conceptos y habilidades que el alumnado deberá manejar durante la actividad; la novedad geográfica atiende al lugar donde se desarrollará la SC; y la novedad psicológica refleja la brecha existente entre las expectativas del alumnado y la realidad a la que se van a enfrentar en el evento.

(2) En la segunda fase es donde tiene lugar la SC, la cual debe concebirse como una parte más del plan de estudios y no como una actividad aislada. Los objetivos de esta etapa van dirigidos a: comprender e investigar aquellas cuestiones relacionadas con el fenómeno científico estudiado. Por tanto, el rol del docente debería ser el de moderador.

(3) La tercera y última fase tiene por objetivo reflexionar sobre la SC en aras de dar respuesta a las intrigas y dudas que surgieran en el alumnado.

Consecuentemente, la SC conlleva un trabajo previo y posterior que podría desarrollarse en el aula, de este modo las actividades desarrolladas fuera del aula se convertirían en un complemento o refuerzo al proceso de enseñanza-aprendizaje que tiene lugar en la escuela.

\section{La enseñanza-aprendizaje de las ciencias ¿necesita salir del aula?}

Casi con total seguridad, si preguntásemos esto a cualquier docente o académico del área de DCE daría una respuesta afirmativa y, seguramente, muchos coincidirían en las ventajas que supone salir del aula tanto como en las limitaciones. Sin embargo, la realidad no es otra sino que las salidas de campo son actividades esporádicas y, habitualmente, desvinculadas del plan de estudios. A pesar de que algunas instituciones (National Research Council 2001) e informes de investigación, tanto internacionales (Informe Rocard, Rocard 2007) como nacionales (Informe ENCIENDE, COSCE 2011) realizan recomendaciones que apuntan a una enseñanza de las ciencias donde el alumno pueda aplicar el conocimiento adquirido y relacionarlo con la vida real, de forma que este pueda ser percibido como útil para su vida cotidiana, colocándose las SC como un recurso didáctico adecuado para tales intenciones. La aplicación de ello contribuiría a mejorar la percepción que tiene el alumnado de las asignaturas de ciencias, generalmente poco atrayentes e irrelevantes para su vida diaria (Solbes 2011), situación que podría encontrar justificación en la densidad de los programas didácticos de ciencias, la complejidad del lenguaje científico y el olvido por parte del profesorado de los intereses del alumnado (Cañas y Martín-Díaz 2010). 
Desde otra perspectiva, alejándonos de suposiciones y acercándonos a los resultados de la investigación educativa, la necesidad de salir del aula para enseñar y aprender ciencias queda patente al confirmarse que el gusto mostrado por el alumnado hacia las ciencias está directamente relacionado con la realización de SC (Kiesel 2005) y, que a su vez, éste se siente más predispuesto y motivado cuando se realizan experiencias fuera del aula (Dillon et al. 2006). Así, es necesario resaltar la influencia de las SC en aspectos de índole emocional, conceptual y procedimental, pues posibilita la observación, la indagación y la discusión (Del Toro y Morcillo 2011).

Al hilo de lo anterior, Eshach (2007) afirma que el alumnado disfruta durante las SC y que es consciente del objetivo didáctico de estas actividades, por lo que no se trata de un «día de diversión» sino más bien de un día en el que «se aprende ciencia de forma divertida». A esto, Rickinson et al. (2004) agregan que las SC tienen un impacto positivo en: (1) las actitudes, creencias y autopercepción del alumnado; y (2) las habilidades sociales, tales como las habilidades de comunicación, cooperación y empatía. Por tanto, nos encontramos que la actitud del alumnado hacia las SC es positiva, dado que al presentar el conocimiento estudiado contextualizado en el mundo real, se permite la aplicación del mismo y se realza su utilidad (Braund y Reiss 2006). Asimismo, esta situación contrasta con la actitud del alumnado hacia la ciencia cada vez más deteriorada, de este modo las SC se colocan como una oportunidad para mejorarla (Rebar y Enochs 2010).

En definitiva, tener en cuenta este recurso didáctico significaría no seguir posponiendo recomendaciones didácticas como la emitida por John Dewey:

We cannot overlook the importance for educational purposes of the close and intimate acquaintance got with nature at first hand, with real things and materials, with the actual processes of their manipulation, and the knowledge of their social necessities and uses... Verbal memory can be trained in committing tasks, a certain discipline of the reasoning powers can be acquired through lessons in science and mathematics; but, after all, this is somewhat remote and shadowy compared with the training of attention and of judgment that is acquired in having to do with things with real motive behind and a real outcome ahead (Dewey 2008, pp. 10-11).

\section{Método}

El método empleado en este trabajo ha sido el de revisión sistemática, presentándose este como una herramienta objetiva, rigurosa y eficaz para un proceso de revisión de la literatura científica sobre un campo de conocimiento concreto y capaz de sintetizar las evidencias empíricas sobre el mismo (Hunt, 1997).

\section{Procedimiento de selección de trabajos y extracción de datos}

El proceso de selección de los artículos se ha desarrollado según las fases propuestas por Sánchez-Meca y Botella (2010):

(1) Formulación de las preguntas de investigación.

(2) Definición de los criterios de selección.

(3) Ejecución de la búsqueda bibliográfica.

De este modo, tras decidir las preguntas de investigación a las que pretende responder este estudio (mostradas en la introducción), se establecieron cuatro criterios para seleccionar los trabajos que compondrían más tarde la muestra de este estudio.

(1) Según el tipo de documento, solo se consideran los artículos. 
(2) Según la fecha de publicación, se contemplan los publicados entre el 2000 y el 2017.

(3) Según la lectura de su título, resumen y palabras clave, se tienen en cuenta los trabajos que utilizan el término salida de campo (field trip, en inglés) y que están enmarcados en el área de DCE.

(4) Según la lectura completa de los trabajos, se incluyen los artículos que aportan implicaciones educativas referentes a las SC (quedaron excluidos aquellos trabajos que versan sobre SC virtuales).

Una vez establecidos los criterios de selección, se realizó la búsqueda bibliográfica en la primera quincena de diciembre de 2017 en las bases de datos electrónicas Web of Science (WOS), restringiendo la búsqueda a su colección principal, y Scopus. Las palabras clave empleadas fueron «field trip» y «science education» unidas a través del operador booleano AND. La búsqueda inicial evidenció una población de 531 trabajos al introducir las palabras clave, de esta forma la muestra se seleccionó a partir de la aplicación sucesiva de los cuatro criterios de inclusión (Tabla 1).

Tabla 1. Proceso de selección según los criterios de inclusión.

\begin{tabular}{llllll}
\cline { 1 - 2 } Fases de la búsqueda & WOS & Scopus & Total & Porcentaje* \\
\cline { 1 - 3 } \cline { 5 - 6 } Búsqueda inicial & 179 & 352 & 531 & \\
Criterio 1 & 118 & 218 & 336 & $63.3 \%$ \\
Criterio 2 & 113 & 181 & 294 & $55.4 \%$ \\
Criterio 3 & 35 & 56 & 91 & $17.1 \%$ \\
Criterio 4 & 18 & $34\left(19^{\mathrm{a}}\right)$ & $52\left(37^{\mathrm{a}}\right)$ & $9.8 \%$ \\
\hline
\end{tabular}

* Porcentaje de trabajos seleccionados respecto a la búsqueda inicial.

${ }^{a}$ Número de artículos al suprimir las coincidencias halladas entre ambas bases de datos.

La aplicación de los criterios de inclusión significó la selección de 52 artículos científicos que representan el 9.8\% de aquellos obtenidos en la búsqueda inicial. Finalmente, se eliminaron las 15 coincidencias detectadas entre los resultados de búsqueda en ambas bases de datos, de esta forma la muestra de estudio asciende a 37 artículos (Anexo).

Respecto a la extracción de datos, la muestra objeto de estudio se analizó conforme a los siguientes ítems: (a) autor/es y año, (b) disciplina científica, (c) país, (d) etapa educativa, (e) contexto, (f) metodología e (g) implicaciones educativas. En cuanto a los procedimientos de extracción de datos seguidos hemos de destacar que:

(1) Los ítems $a, c$ y $d$ se identificaron a partir de la lectura de los artículos seleccionados, pues se trata de datos objetivos que no dan lugar a interpretación.

(2) Los ítems $b$ y $f$ se analizaron en cada trabajo a través de un sistema de categorías deductivo, elaborado a partir de distintos marcos teóricos de referencia (propios de la Ciencia y de la metodología de investigación). Así, para el ítem $b$ se optó por establecer las categorías: Medio ambiente, Biología, Geología, Química y Física, y para el ítem $f$ se establecieron las categorías: Metodología cuantitativa, cualitativa y método mixto.

(3) Por último, los ítems e y $g$ se analizaron conforme a un sistema de categorías inductivo, diseñado a partir de la lectura de los artículos seleccionados. De modo que para clasificar el contexto de ejecución de las SC se agruparon en una única categoría aquellos de características similares (como es el caso de los espacios naturales y urbanos) y se establecieron como categorías independientes los más singulares (entiéndanse: museo, planetario, acuario...).

En cuanto a las limitaciones, hemos de atender a la definición dada por Krepel y Durral (1981) de SC, donde colocan como sinónimos a los términos excursion escolar (school excursión), 
itinerario didáctico (instructional trip) y jornada escolar (school journey). Así, a la vez que representa la principal limitación de este trabajo (solo se ha empleado la palabra clave «field trip» cruzada con «science education»), también evoca a la realización de futuros estudios de revisión donde se crucen todas las palabras clave mencionadas.

\section{Resultados y Discusión}

\section{Evolución de la producción científica en el siglo XXI}

Al comparar el número de artículos seleccionados en este estudio con aquel obtenido en revisiones precedentes, como la realizada por Mason (1981), se puede observar que la evolución de la producción científica que aborda la SC como un recurso didáctico ha aumentado considerablemente. Este incremento respecto al siglo pasado, queda reflejado en 43 trabajos incluidos dentro de un lapso de tiempo de unos 60 años, mientras que desde el año 2000 hasta el 2017 se han publicado prácticamente una cuarentena de artículos sobre esta temática.

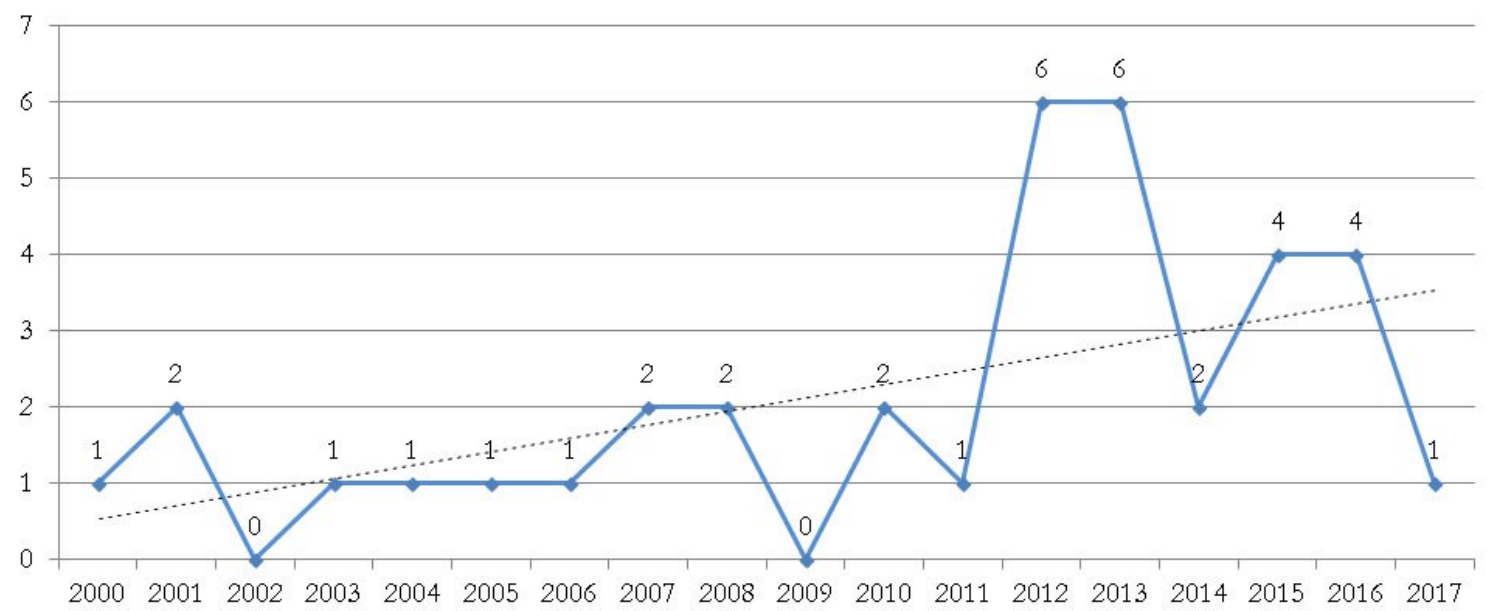

Figura 1. Evolución de la producción científica desde el 2000 hasta el 2017.

Tal y como se puede ver en la Figura 1, el número de artículos seleccionados por año es escaso, más si tenemos en cuenta que los datos expuestos son la suma de aquellos artículos seleccionados entre las bases de datos WOS y Scopus. Este hecho puede ser fruto de la escasez de experiencias educativas relativas a las SC (Pedrinaci 2012). No obstante, podemos observar que la tendencia de publicación de artículos sobre esta línea de investigación es positiva, ya que el $62 \%(\mathrm{n}=23)$ de los trabajos seleccionados han sido publicados entre el 2012 y el 2017. Además, se ha identificado una correlación moderada entre el año de publicación y el número de artículos publicados $\left(\mathrm{r}=0.524, \mathrm{p} .<0.05, \mathrm{r}^{2}=0.28\right)$.

\section{Autores prolíficos, índice de colaboración y grupos de investigación}

Se han identificado 84 autores entre los 37 artículos seleccionados, dato que arroja un indice de colaboración de 2.3 autores por trabajo. Este resultado es ligeramente superior al promedio de dos autores por trabajo obtenido en la rama de Ciencias Sociales en España y claramente superior al 1.81 obtenido para el área de DCE (Anta y Pérez 2007). Este hecho puede deberse al esfuerzo que deben realizar los docentes y demás partes implicadas en el diseño y puesta en marcha de una SC (Rebelo et al. 2011), por lo que, al existir un mayor número de personas implicadas, es lógico que el índice de colaboración en la publicación de los trabajos aumente. 
$\mathrm{Al}$ analizar las autorías de cada trabajo, se han identificado ocho artículos de un único autor lo que representa el 21.6\% de la muestra, así como que el 96.4\% de los autores ( $n=81$ ) solo dispone de un artículo publicado en el lapso temporal analizado (Tabla 2). Por otro lado, solamente el 3.6\% de los autores tienen dos o más trabajos publicados sobre SC. En este sentido, podría afirmarse que no son muchos los autores que tienen una gran producción científica en esta línea de investigación dado que la autora más productiva es R.T. Tal con seis publicaciones, seguida de O. Morag con cinco publicaciones.

Tabla 2. Relación de autores y número de trabajos publicados sobre SC.

\begin{tabular}{lcc}
\hline & $\mathbf{N}^{\mathbf{o}}$ de autores & Porcentaje \\
\hline Autores con 1 trabajo & 81 & $96.4 \%$ \\
Autores con 2 trabajos & 1 & $1.2 \%$ \\
Autores con 3 o más trabajos & 2 & $2.4 \%$ \\
\hline
\end{tabular}

En cuanto a la identificación de grupos de investigación, parece que el escaso número de autores prolíficos en esta línea de investigación es determinante en el número de posibles grupos de investigación. Así, siguiendo las directrices de Peiró (1981), en las que se considera que un grupo de investigación queda constituido por aquellos autores que colaboran entre sí y todos aquellos que han colaborado con alguno de estos, adoptando como criterio mínimo que un grupo esté formado por tres autores y más de tres artículos. De este modo, se ha encontrado un grupo de investigación formado por cinco autores (R.T. Tal, O. Moral, Y.J. Dori, Y. Bamberger y N. Lavie Alon) que han aportado seis publicaciones, del cual R.T. Tal es la autora principal dado que participa en los seis artículos, cuatro de ellos como primera autora.

\section{Países, etapas educativas, disciplinas científicas y contextos donde se desarrollan las investigaciones}

Países. Los trabajos seleccionados han sido desarrollados en 13 países diferentes (Tabla 3) entre los que destacan Estados Unidos (17), Israel (6), Portugal (3) y Alemania (2). Además de los países citados, se encontró un trabajo en: Turquía, Nueva Zelanda, Malasia, España, Dinamarca, Inglaterra, Canadá, Eslovenia y Eslovaquia. La existencia de esta amplia gama de países donde se desarrollan trabajos de investigación relacionados con las SC, aunque no todos con la misma intensidad, tiene que ver con la aceptación general por parte de la investigación educativa y del profesorado de las bondades de las SC como recurso educativo (Orion 1993).

Tabla 3. Países donde se desarrollan los trabajos sobre SC.

\begin{tabular}{ccc}
\hline País & Frecuencia & Porcentaje \\
\hline Estados Unidos & 17 & $46 \%$ \\
Israel & 6 & $16.2 \%$ \\
Portugal & 3 & $8.1 \%$ \\
Alemania & 2 & $5.4 \%$ \\
Turquía & 1 & $2.7 \%$ \\
Nueva Zelanda & 1 & $2.7 \%$ \\
Malasia & 1 & $2.7 \%$ \\
España & 1 & $2.7 \%$ \\
Dinamarca & 1 & $2.7 \%$ \\
Inglaterra & 1 & $2.7 \%$ \\
Canadá & 1 & $2.7 \%$ \\
Eslovenia & 1 & $2.7 \%$ \\
Eslovaquia & 1 & $2.7 \%$ \\
\hline Total & 37 & $100 \%$ \\
\hline
\end{tabular}

Respecto al caso de España, la publicación de artículos sobre SC enmarcados en el área de DCE es muy baja. Esto podría guardar relación directa con la legislación educativa vigente en 
nuestro país (Ley Orgánica para la Mejora de la Calidad Educativa, LOMCE) en la cual se valora escasamente el cómo enseñar Ciencia (Fernandes, Pires y Delgado-Iglesias 2018).

Etapas educativas (Tabla 4). Los artículos seleccionados se han llevado a cabo en las etapas educativas de Educación Primaria (13), Educación Secundaria (8), Universidad (10) y Egresados, en relación a la formación continua del profesorado (4). La etapa de Educación Infantil queda sin representación entre los artículos seleccionados, colocándose una vez más como la gran olvidada en DCE (Pro y Rodríguez 2010). Opuesto es el caso de la Educación Primaria que abarca el 35.1\% de la muestra, coincidiendo ello con DeWitt y Storksdiek (2008) quienes resaltan el número de investigaciones sobre SC destinadas a esta etapa educativa.

Tabla 4. Etapas educativas donde se desarrollan los trabajos sobre SC.

\begin{tabular}{ccc}
\hline Etapa educativa & Frecuencia & Porcentaje \\
\cline { 2 - 3 } Educación Primaria & 13 & $35.1 \%$ \\
Educación Secundaria & 8 & $21.6 \%$ \\
Universidad & 10 & $27 \%$ \\
Egresados & 4 & $10.8 \%$ \\
No procede & 2 & $5.4 \%$ \\
Total & 37 & $100 \%$ \\
\hline
\end{tabular}

Por otro lado, la Tabla 4 muestra dos trabajos clasificados como «no procede» etapa educativa, estos son los estudios 29 y 30 (según la enumeración expuesta en el Anexo) cuyos objetivos atienden a evaluar la calidad de distintas SC.

Disciplinas cientificas. Entre los trabajos seleccionados se abarcan las disciplinas científicas de Biología, Geología, Física, Química y Medio Ambiente. Las disciplinas más destacadas, véase la Tabla 5, son: Medio Ambiente (11), Biología (10) y Geología (10). Ello responde al habitual reconocimiento de las SC como un recurso didáctico propio de la Biología y la Geología (Costillo, Cañada, Conde y Cubero 2011; Orion 1993). No obstante, dada la necesidad de formar a la ciudadanía en materia medio ambiental en aras de crear conciencia y conductas responsables para cuidar el medio, el número de SC destinadas a esta disciplina científica ha crecido hasta el punto de ser la más recurrente.

El total de clasificaciones realizadas respecto a la disciplina asciende a 40 , esto se debe a que los estudios 2, 8, 14 y 18 abarcan dos disciplinas científicas y el trabajo 37 se ocupa de tres disciplinas diferentes. Contrario a esto, el artículo 28 no especifica la disciplina abordada y en los estudios 29 y 30 no procede la clasificación en ninguna disciplina científica, dado que son trabajos destinados a la evaluación del diseño de varias SC.

Tabla 5. Disciplinas científicas en las que se realizan las SC.

\begin{tabular}{ccc} 
Tabla 5. Disciplinas científicas en las que se realizan las SC. \\
\hline Disciplina & Frecuencia & Porcentaje \\
\cline { 3 - 3 } Medio Ambiente & 11 & $27.5 \%$ \\
Biología & 10 & $25 \%$ \\
Geología & 10 & $25 \%$ \\
Química & 5 & $12.5 \%$ \\
Física & 3 & $7.5 \%$ \\
No especifica & 1 & $2.5 \%$ \\
No procede & 2 & $5.4 \%$ \\
Total & 40 & $100 \%$ \\
\hline
\end{tabular}

Contextos. Las SC analizadas se realizan mayormente en espacios naturales (25.5\%), como puede observarse en la Tabla 6. También, son frecuentes las SC destinadas a visitar museos y centros de investigación (23.2\% entre ambos lugares), sobre ello Michie (1998) puntualiza que el mayor volumen de la literatura sobre esta línea de investigación proviene de las SC realizadas en museos y centros de investigación. Sin embargo, parece que actualmente la 
tendencia ha cambiado a favor de los espacios naturales, lo que podría estar ligado a la irrupción de la disciplina científica de Medio Ambiente. Así, se han obtenido 43 clasificaciones según los contextos abordados, de forma que los estudios 16 y 17 visitaron dos contextos diferentes y los estudios 23 y 26 asistieron a tres y cuatro contextos distintos respectivamente. Además, se han identificado dos estudios que no identifican los contextos visitados (trabajos 7 y 31) y tres artículos donde no procede clasificarlos según el contexto, pues los estudios 29 y 30 se encargan de evaluar la calidad del diseño de varias SC sin experimentar la visita a ningún contexto, mientras que el estudio 36 analiza la predisposición del profesorado hacia la puesta en marcha de SC en la disciplina científica de Geología.

Tabla 6. Contextos en los que se realizan las SC.

\begin{tabular}{ccc}
\hline Contexto & Frecuencia & Porcentaje \\
\hline Espacio natural & 11 & $25.5 \%$ \\
Museo & 5 & $11.6 \%$ \\
Centro de investigación & 5 & $11.6 \%$ \\
Espacio urbano & 4 & $9.3 \%$ \\
Zoológico & 3 & $7 \%$ \\
Universidad/Facultad & 2 & $4.7 \%$ \\
Aula de naturaleza & 2 & $4.7 \%$ \\
Empresas & 2 & $4.7 \%$ \\
Acuario & 1 & $2.3 \%$ \\
Depuradora & 1 & $2.3 \%$ \\
Planetario & 1 & $2.3 \%$ \\
Central nuclear & 1 & $2.3 \%$ \\
No especifica & 2 & $4.7 \%$ \\
No procede & 3 & $7 \%$ \\
Total & 43 & $100 \%$ \\
\hline
\end{tabular}

A la luz de los resultados obtenidos al cruzar las variables disciplina científica y contexto, se ha identificado que el 40\% de los trabajos que abordan la disciplina de Biología, el 50\% de los de Geología y el 36\% de los de Medio Ambiente desarrollan sus SC en espacios naturales (tales como: ríos, estanques, bosques...), siendo en estas disciplinas el contexto más visitado. En cuanto a los estudios sobre las disciplinas de Física y Química, estos prefieren realizar sus SC a centros de investigación, pues así ha ocurrido en el $66 \%$ y $40 \%$ de los trabajos que abordan estas disciplinas respectivamente. Sin embargo, se ha comprobado que el contexto más versátil para realizar SC respecto a la disciplina científica abordada es el aula de naturaleza, puesto que en este contexto se han trabajado las disciplinas de Biología, Geología, Química y Medio Ambiente.

\section{Metodologías de investigación}

La Tabla 7 recoge las frecuencias y porcentajes de aquellos estudios que usan la metodología cuantitativa, cualitativa o método mixto. Asimismo, se ha encontrado cierta preferencia por el empleo de la metodología cualitativa, pues el $40.5 \%$ de los estudios seleccionados la utiliza.

Tabla 7. Metodologías empleadas en los artículos referidos a SC.

\begin{tabular}{ccc}
\hline Metodología & Frecuencia & Porcentaje \\
\hline Metodología cuantitativa & 9 & $24.3 \%$ \\
Metodología cualitativa & 15 & $40.5 \%$ \\
Método mixto & 12 & $32.4 \%$ \\
No especifica & 1 & $2.7 \%$ \\
Total & 37 & $100 \%$ \\
\hline
\end{tabular}

Este hecho contrasta con lo enunciado por Michie (1998), quien afirma que la mayoría de los trabajos relacionados con las SC emplean métodos cuantitativos. Por tanto, podría afirmarse que en esta línea de investigación se ha producido un cambio metodológico importante puesto 
que entre aquellos artículos que emplean una metodología cualitativa y aquellos que usan un método mixto representan casi el 73\% de la muestra. En este sentido, Amórtegui et al. (2016) coinciden, en su revisión, al resaltar la preferencia de los investigadores por métodos de carácter cualitativo para estudiar las concepciones y los conocimientos del profesorado sobre las SC. Ello puede responder a la tendencia expuesta por Gutiérrez (2008) en lo que al empleo de metodologías de la investigación se refiere en Didáctica de las Ciencias Experimentales, y a las bondades que la metodología cualitativa ofrece al investigador que intenta acceder a las percepciones, emociones u otras cuestiones que necesitan del diálogo y la observación.

\section{Implicaciones educativas}

La Tabla 8 muestra la síntesis de aquellas implicaciones educativas sobre las SC aportadas por los artículos seleccionados, de forma que se han agrupado por núcleos temáticos y se han asociado al número asignado a cada artículo seleccionado según el Anexo.

Respecto a las cinco categorías establecidas para clasificar las implicaciones educativas, entiéndanse: (1) emociones, motivación y actitudes; (2) adquisición y transferencia del conocimiento; (3) imagen de la ciencia; (4) factores que inciden en la calidad y el éxito de la SC; y (5) factores que limitan la realización de SC, se ha obtenido que el 76\% (n $=28)$ de los artículos seleccionados aportan implicaciones educativas referentes a la primera categoría y/o segunda. Este hecho coincide con lo expuesto por Michie (1998), al afirmar que la mayoría de estudios ligados a las SC abordan el logro académico y las actitudes del alumnado.

Tabla 8. Síntesis de las implicaciones educativas relativas a SC.

\begin{tabular}{|c|c|}
\hline Referidas a & Implicaciones educativas \\
\hline $\begin{array}{c}\text { Emociones, } \\
\text { motivación y } \\
\text { actitudes }\end{array}$ & $\begin{array}{ll}\text { - } & \text { Incrementa el interés y la motivación por aprender ciencias }(1,4,6,8,10,11,16,17,23, \\
& 26,27,33,37) . \\
\text { - } & \text { No mejora el interés por las ciencias del alumnado }(15,35) . \\
\text { - } & \text { Promueven el interés por estudiar carreras científicas }(17,26) . \\
\text { - } & \text { Mejora la autoeficacia del alumnado (9). } \\
\text { - } & \text { Genera emociones positivas durante el aprendizaje de las ciencias (32). }\end{array}$ \\
\hline $\begin{array}{c}\text { Adquisición y } \\
\text { transferencia } \\
\text { del } \\
\text { conocimiento }\end{array}$ & $\begin{array}{ll}\text { - } & \text { Mejora la adquisición y comprensión de los contenidos científicos }(1,3,6,8,9,11,15,18, \\
\text { - } & \text { Eleva el rendimiento académico }(26,34) . \\
\text { - } & \text { Vincula los contenidos trabajados en el aula con el mundo real, permitiendo su aplicación } \\
& \text { y probando su utilidad }(2,3,7,11,13,16,19,21,23,24,33,35) . \\
\text { - } & \text { Proporciona un aprendizaje experiencial }(1,19,22) . \\
\text { - } & \text { Contribuye al aprendizaje significativo duradero en el tiempo }(1,10,21,27,32,37) . \\
\text { - } & \text { Promueve un ambiente adecuado para el aprendizaje de las ciencias }(3,8,23) . \\
\text { - } & \text { Fomenta la reflexión y el trabajo grupal }(7,18,27) .\end{array}$ \\
\hline $\begin{array}{l}\text { Imagen de la } \\
\text { ciencia }\end{array}$ & - $\quad$ Mejora las creencias del alumnado sobre la ciencia y su opinión hacia los científicos (26). \\
\hline
\end{tabular}

La contribución de la SC en el ámbito emocional, motivacional y social del aprendizaje es evidente según los resultados obtenidos. Ello también lo comprobaron Behrendt y Franklin (2014) al manifestar que la SC es una excelente oportunidad para motivar al alumnado hacia el aprendizaje de las ciencias. Asimismo, Hutson, Cooper y Talbert (2011) afirman que la SC podría impactar de forma positiva en el interés y en la futura decisión del alumnado por estudiar una carrera científica. Además, se han encontrado aportaciones que apuntan a la promoción de emociones positivas durante el aprendizaje de las ciencias (estudio 32), ello podría revertir que el alumnado experimente de forma habitual emociones negativas como el aburrimiento, el nerviosismo y la preocupación durante el aprendizaje de la Física y la Química (Dávila-Acedo 2017), dado que la forma de dar clase, el contenido abordado y la falta de trabajo práctico pueden ser los detonantes de las experiencias emocionalmente negativas del alumnado hacia el aprendizaje de las ciencias (Daschmann, Goetz y Stupnisky 2014). 
Sin embargo, se han identificado dos estudios (15 y 35) que no obtuvieron mejoras respecto al interés del alumnado por aprender ciencias. La razón de esto puede atender a los detalles que estos mismos trabajos aportan, pues el alumnado participante partía con unos niveles considerables en lo que interés por las ciencias se refiere. Por tanto, parece oportuno aludir al «efecto techo» para justificar dichos resultados, al menos en esta ocasión.

En cuanto al aspecto cognitivo del aprendizaje, los resultados obtenidos vuelven a ser meridianos. Así, la SC se convierte en una herramienta didáctica que mejora la comprensión de los contenidos e incrementa el conocimiento del alumnado (Orion 1993; Orion 2007). Ello posibilitado por el aprendizaje experiencial (Lei 2010) y la conexión de lo aprendido con el mundo real (Behrendt y Franklin 2014), junto con el ambiente adecuado para aprender ciencias, el trabajo cooperativo y los procesos reflexivos generados por la SC.

Tabla 8. Continuación

\begin{tabular}{|c|c|}
\hline Referidas a & Implicaciones educativas \\
\hline $\begin{array}{c}\text { Factores que } \\
\text { inciden en la } \\
\text { calidad y el } \\
\text { éxito de la SC }\end{array}$ & $\begin{array}{ll}\text { - } & \text { El tiempo del que disponen los docentes para la preparación y ejecución de la SC }(25,30, \\
& 33,35) \text {. } \\
\text { - } & \text { La coordinación entre todas las partes implicadas (docentes, guías, padres...) }(9,20,22, \\
& \text { 25, 29, 30). } \\
\text { - } & \text { El grado de implicación del docente }(25,29,28,30) \text {. } \\
\text { - } & \text { La formulación de unos objetivos claros y concisos }(12,20,29) \text {. } \\
\text { - } & \text { La estimulación de la participación activa del alumnado }(4,6,29,30) \text {. } \\
\text { - } & \text { La formación del profesorado sobre la gestión, el diseño y la ejecución de SC }(14,22) \text {. } \\
\text { - } & \text { El compromiso de los organizadores de la SC influye en el nivel de compromiso, actitud y } \\
\text { - } & \text { motivación del alumnado (21). } \\
\text { - } & \text { La conexión de la SC con el currículum }(10,20,27) \text {. } \\
\text { institución educativa (5). } \\
\text { - } \quad \text { La metodología de enseñanza empleada, alejándose esta de las tradicionales }(10,30,33) \text {. }\end{array}$ \\
\hline $\begin{array}{l}\text { Factores que } \\
\text { limitan la } \\
\text { realización de } \\
\text { SC }\end{array}$ & $\begin{array}{l}\text { - La realización de SC depende de las experiencias anteriores del profesorado y de su } \\
\text { conexión con el temario (36). } \\
\text { - Las principales razones por las que el profesorado decide no realizar SC son: falta de } \\
\text { recursos y de formación (36). } \\
\text { - } \quad \text { La burocracia y la excesiva carga laboral de los docentes (31). } \\
\text { - } \quad \text { Se necesita una supervisión exhaustiva al realizarse fuera del recinto escolar (4). }\end{array}$ \\
\hline
\end{tabular}

Finalmente, se han hallado algunos factores que pudieran determinar la calidad y el éxito de una SC, e incluso limitar su práctica. En este sentido, Jarvis y Pell (2005) confirman que el compromiso y el entusiasmo hacia la ciencia del profesorado está estrechamente relacionado con la promoción de actitudes positivas en el alumnado durante una SC. También, se alude a la formación y experiencia del profesorado sobre SC (Behrendt y Franklin 2014), la preparación de la SC (coordinación, implicación y objetivos) y a la inclusión de este tipo de actividades en el plan de estudios para conseguir una SC de calidad y exitosa (Orion y Hofstein 1994). Además, el éxito de la SC precisa de un enfoque didáctico en el que se promueva la participación del alumnado (Behrendt y Franklin 2014), la iniciativa y la indagación, así como reducir la cantidad de información a transmitir durante dicha actividad (Roldán, Ulloa, Vargas, Chura y Pacheco 2017). Por otro lado, aquellos factores limitantes encontrados están muy relacionados con la percepción del profesorado sobre las SC, pues las consideran un trabajo y una dedicación extra (Rebar y Enochs 2010) y por la situación laboral que les envuelve, como son: el número de alumnos por aula, los aspectos económicos y burocráticos, la escasez de materiales didácticos sobre estas actividades y la responsabilidad civil (Rebelo et al. 2011). 


\section{Conclusiones}

La revisión de la literatura realizada aporta, por un lado, el desarrollo de la línea de investigación que forma el estudio de las SC como recurso didáctico en el área de DCE; y por otro, la síntesis de las principales implicaciones educativas. De este modo, respecto a las preguntas de investigación enunciadas, podemos concluir que:

Pregunta 1. La producción científica sobre SC en el periodo temporal analizado (2000-2017) tiene una tendencia positiva, en consecuencia, más de la mitad de los artículos seleccionados en esta revisión sistemática han sido publicados entre 2012 y 2017. Por tanto, parece que la investigación sobre las SC como recurso didáctico en DCE es incipiente.

Pregunta 2. A pesar de existir una amplia relación de autores que ostentan publicaciones sobre la línea de investigación considerada, el número de autores con una producción considerable es escaso. En este sentido, R.T. Tal y O. Morag se sitúan como las autoras más prolíficas. Tan es así, que a partir de la autora R.T. Tal se configura el único grupo de investigación identificado. No obstante, el grado de colaboración obtenido es superior al índice medio calculado para la DCE, pudiendo concluir que la investigación sobre SC requiere mayor grado de colaboración que otras líneas de investigación.

Pregunta 3. El número de países donde se llevan a cabo los trabajos es amplio, pero entre ellos resaltan Estados Unidos e Israel, lo cual los sitúa como pioneros en esta línea de investigación. Respecto a la etapa educativa, una vez más podemos asentir que la Educación Infantil no suele ser considerada para las investigaciones de DCE, mientras que la Educación Primaria ha sido la etapa a la que más investigaciones se le han dedicado. Además, este estudio vuelve a confirmar la preferencia de las SC como recurso didáctico para enseñar Biología y Geología. Aunque, la cifra de trabajos destinados a abordar el Medio Ambiente es la mayor, hecho que podría ser indicador de la importancia de concienciar al alumnado sobre las acuciantes problemáticas medio ambientales. En cuanto al contexto, se puede alegar que el contexto más frecuente para desarrollar una SC es el medio natural, pero las aulas de naturaleza parecen el contexto más versátil para trabajar las distintas disciplinas científicas.

Pregunta 4. Se podría sostener que en el siglo XXI se ha producido un cambio metodológico en la investigación en DCE, o al menos en la línea de investigación estudiada, pues la metodología cualitativa ha sustituido a la cuantitativa como la más utilizada.

Pregunta 5. Las implicaciones educativas identificadas esbozan un panorama alentador para la implementación de las SC como recurso didáctico para enseñar ciencias, dados los beneficios aportados en aspectos motivacionales y afectivos, como en los cognitivos que componen el proceso de enseñanza-aprendizaje desarrollado entre el binomio alumno-profesor. No obstante, se ha de prestar especial atención a aquellos factores que pudieran incidir en el desarrollo de la SC, tales como: el compromiso y la formación del profesorado, los quehaceres burocráticos, la metodología de enseñanza empleada y rol otorgado al alumnado.

Finalmente, a la luz de los resultados, parece oportuno abogar por un cambio en la consideración de las SC, tanto desde la perspectiva del profesorado como de la política educativa. Asimismo, sería conveniente que las SC pasaran de ser una actividad extra-curricular y esporádica a ser incluidas en el marco curricular y consideradas como un recurso didáctico adecuado y efectivo para enseñar ciencias.

\section{Referencias}

Allen S. (2004) Designs for Learning: Studying Science Museum Exhibits That Do More Than Entertain. Science Education 88(1), 17-33. 
Álvarez-Piñeros D., Vásquez-Ortiz W.F., Rodríguez-Pizzinato L.A. (2016) La salida de campo, una posibilidad en la formación inicial docente. Didáctica de las Ciencias Experimentales y Sociales 31, 61-78.

Amórtegui E., Gavidia V., Mayoral O. (2016) Las prácticas de campo en la enseñanza de la Biología y la formación docente: Estado actual del conocimiento. Actas de los XXVII Encuentros de Didáctica de las Ciencias Experimentales (7-9 de septiembre) 175-182. Badajoz, España. Recuperado el 12 de abril de 2018 de http://www.apicedce.com/sites/default/files/XXVII\%20Actas.pdf

Anta C., Pérez J.M. (2007) La producción cientifica sobre Didáctica de las Ciencias. IV Congreso de Comunicación Social de la Ciencia. Madrid: CSIC.

Behrendt M., Franklin T. (2014) A Review of Research on School Field Trips and Their Value in Education. International Journal of Environmental \& Science Education 9, 235-245.

Braund M., Reiss M. (2006) Towards a more authentic science curriculum: The contribution of out-of-school learning. International Journal of Science Education 28(12), 1373-1388.

Cañas A., Martín-Díaz M.J. (2010) ¿Puede la competencia científica acercar la ciencia a los intereses del alumnado? Alambique 66, 80-87.

COSCE-Confederación de Sociedades Científicas de España (2011) Informe ENCIENDE. Enseñanza de las Ciencias en la Didáctica Escolar para edades tempranas en España. Madrid: COSCE. Recuperado el 29 de noviembre de 2017, de http://www.cosce.org/pdf/Informe_ENCIENDE.pdf

Costillo E., Cañada F., Conde M.C., Cubero J. (2011) Conceptions of prospective teachers on nature field trips in relation to own experiences as pupils. 9th Conference of the European Science Education Research Association. Lyon: Francia.

Daschmann E., Goetza T., Stupnisky R. (2014) Exploring the antecedents of boredom: Do teachers know why students are bored? Teaching and Teacher Education 39, 22-30.

Dávila-Acedo M.A. (2017). Las emociones y sus causas en el aprendizaje de Física y Química, en el alumnado de Educación Secundaria. Revista Eureka sobre Enseñanza y Divulgación de las Ciencias 14(3), 570-586.

Del Toro R., Morcillo J.G. (2011) Las actividades de campo en educación secundaria. Un estudio comparativo entre Dinamarca y España. Enseñanza de las Ciencias de la Tierra 19(1), 39-47.

Dewey J. (2008) The school and society. Delhi: AAKAR Books.

DeWitt J., Storksdieck M. (2008) A Short Review of School Field Trips: Key Findings from the Past and Implications for the Future. Visitor Studies 11(2), 181-197.

Dillon J., Rickinson M., Teamey K., Morris M., Choi M.Y., Sanders D., Benefield, P. (2006) The value of outdoor learning: evidence from research in the UK and elsewhere. School Science Review 87, 107-111.

Eshach H. (2007) Bridging in-school and out-of-school learning: Formal, non-formal, and informal learning. Journal of Science Education and Technology 16(2), 171-190.

Fernandes I.M.B., Pires D.M., Delgado-Iglesias J. (2018) ¿Qué mejoras se han alcanzado respecto a la Educación Científica desde el enfoque Ciencia-Tecnología-SociedadAmbiente en el nuevo Currículo Oficial de la LOMCE de $5^{\circ}$ y $6^{\circ}$ curso de Primaria en España? Revista Eureka sobre Enseñanza y Divulgación de las Ciencias 15(1), 1101. 
Gair N.P. (1997) Outdoor education. Theory and Practice. London and Wellington: Cassel.

Guisasola J., Morentín M. (2007) ¿Qué papel tienen las visitas escolares a los museos de ciencias en el aprendizaje de las ciencias? Una revisión de las investigaciones. Enseñanza de las Ciencias 25(3), 401-414.

Gutiérrez J. (2008) Tendencias metodológicas contemporáneas de la investigación en Didáctica de las Ciencias. Actas del XXIII Encuentros de Didáctica de las Ciencias Experimentales (9-12 de septiembre) 1284-1308. Almería, España. Recuperado el 12 de abril de 2018 de http://www.apicedce.com/sites/default/files/APICEACTAS/23EDCEAlmeria2008.pdf

Hunt M. (1997) How science takes stock: The story of meta-analysis. Nueva York: Russell Sage Foundation.

Hutson T., Cooper S., Talbert, T. (2011) Describing connections between science content and future careers: Implementing Texas curriculum for rural at-risk high school students using purposefully-designed field trips. Rural Educator 31, 37-47.

Jarvis T., Pell A. (2005) Factors influencing elementary school children's attitudes toward science before, during, and after a visit to the UK National Space Centre. Journal of Research in Science Teaching 42(1), 53-83.

Kisiel J. (2005) Understanding elementary teacher motivations for science fieldtrips. Science Education 89(6), 936-955.

Krepel W.J., Durral C.R. (1981) Field trips: A guideline for planning and conducting educational experiences. Washington, DC: National Science Teachers Association.

Lei S.A. (2010) Assessment practices of advanced field ecology courses. Education 130(3), 404415.

Mason J.L. (1980) Annotated bibliography of field trip research. School Science and Mathematics $80(2), 155-166$.

Michie M. (1998) Factors influencing secondary science teachers to organise and conduct field trips. Australian Science Teacher's Journal 44, 43-50.

National Research Council (2001) Inquiry and the National Science Education Standards: A guide for teaching and learning. Washington, DC: National Academy Press.

Orion N. (1993) A model for the development and implementation of field trips as an integral part of the science curriculum. School Science and Mathematics 93(6), 325-331.

Orion N. (2001). A educação em Ciências da Terra: da teoria à prática-implementação de novas estratégias de ensino em diferentes ambientes de aprendizagem, pp. 93-114 en Marques L., Praia, J. (Coords.) Geociências nos currículos dos ensinos básico e secundário. Aveiro: Universidade de Averio.

Orion N. (2007) A Holistic Approach for Science Education for All. Eurasia Journal of Mathematics, Science \& Technology Education 3(2), 99-106.

Orion N., Hofstein A. (1994) Factors that influence learning during a scientific field trip in a natural environment. Journal of Research in Science Teaching 31, 1097-1119.

Pedretti E. (2003) Teaching science, technology, society and environment (STSE) education: Preservice teachers' philosophical and pedagogical landscapes, pp. 219-239 en Zeidler D.L. (Ed.) The role of moral reasoning on socioscientific issues and discourse in science education. Dordrecht: Kluwer Academic Press. 
Pedrinaci E. (2012) Trabajo de campo y aprendizaje de las ciencias. Alambique 71, 81-89.

Peiró J.M. (1981) Colegios invisibles en Psicología, pp. 53-78 en Carpintero H., Peiró J.M. (dirs.), Psicología contemporánea. Valencia: Alfaplus.

Pro A., Rodríguez J. (2010) Aprender competencias en una propuesta para la enseñanza de los circuitos eléctricos en Educación Primaria. Enseñanza de las Ciencias, 28(3), 385-406.

Rebar B.M., Enochs L.G. (2010) Integrating environmental education field trip pedagogy into science teacher preparation, pp. 111-126 en Bodzin A., Klein B.S., Weaver S. (Eds.) The inclusion of environmental education in science teacher education. Netherlands: Springer.

Rebelo D., Marqués L., Costa N. (2011) Actividades en ambientes exteriores al aula en la Educación en Ciencias: contribuciones para su operatividad. Enseñanza de las Ciencias de la Tierra 19(1), 15-25.

Rickinson M., Dillon J., Teamey K., Morris M., Choi M.Y., Sanders D., et al. (2004) A review of research on outdoor learning. Shrewsbury, UK: National Foundation for Educational Research and King's College.

Rocard M. (Chair) (2007) Science Education Now: A Renewed Pedagogy for the Future of Europe. European Commission. Recuperado el 29 de noviembre de 2017, de https://sede.educacion.gob.es/publiventa/PdfServlet?pdf=VP15136.pdf\&area=E

Roldán A.I., Ulloa D., Vargas L., Chura Z., Pacheco L.F. (2017) Comparación entre recorridos guiados tradicionales y recorridos guiados indagatorios en el Museo Nacional de Historia Natural, La Paz-Bolivia. Revista Eureka sobre Enseñanza y Divulgación de las Ciencias 14(2), 367-384.

Sánchez-Meca J., Botella J. (2010) Revisiones sistemáticas y meta-análisis: Herramientas para la práctica profesional. Papeles del Psicólogo 31(1), 7-17.

Solbes J. (2011) ¿Por qué disminuye el alumnado de ciencias? Alambique 67, 53-61.

Sorrentino A.V., Bell P.E. (1970) A comparison of attributed values with empirically determined values of secondary school science field trips. Science Education 54(3), 233236.

Tal R.T., Morag O. (2009) Reflective Practice as a Means for Preparing to Teach Outdoors in an Ecological Garden. Journal of Science Teacher Education 20(3), 245-262. 


\section{Anexo I: Referencias bibliográficas de los artículos seleccionados}

(1)Aksüt P., Doğan N., Bahar, M. (2016) If you change yourself, the world changes: the effect of exhibition on preservice science teachers' views about global climate change. Eurasia Journal of Mathematics, Science \& Technology Education 12(12), 2933-2947.

(2)Aubrecht K.B., Padwa L., Shen X., Bazargan G. (2015) Development and Implementation of a Series of Laboratory Field Trips for Advanced High School Students To Connect Chemistry to Sustainability. Journal of Chemical Education 92(4), 631-637.

(3)Bauerle T.L., Park T.D. (2012) Experiential learning enhances student knowledge retention in the plant sciences. HortTechnology 22(5), 715-718.

(4)Birnbaum S. (2004) Overcoming the limitations of an urban setting through field-based earth systems inquiry. Journal of Geoscience Education 52(5), 407-410.

(5)D'Alessio M.A. (2012) Schoolyard geology as a bridge between urban thinkers and the natural world. Journal of Geoscience Education 60(2), 106-113.

(6)Dohn N.B. (2013) Upper secondary students' situational interest: A case study of the role of a zoo visit in a biology class. International Journal of Science Education 35(16), 2732-2751.

(7)Dori Y.J., Tal R.T. (2000) Formal and informal collaborative projects: Engaging in industry with environmental awareness. Science Education 84(1), 95-113.

(8)Esteves H., Ferreira P., Vasconcelos C., Fernandes I. (2013) Geological fieldwork: A study carried out with Portuguese secondary school students. Journal of Geoscience Education 61(3), 318-325.

(9)Fauzi A., Rizman Z.I. (2015) Field Trip Education Approach Beyond Classroom: Microwave Course Case. Mediterranean Journal of Social Sciences 6(4), 89-94.

(10)Henriques M.H., Tomaz C., Sá A.A. (2012) The Arouca Geopark (Portugal) as an educational resource: a case study. Episodes 35(4), 481-488.

(11)Hudak P.E. (2003) Campus field exercises for introductory geoscience courses. Journal of Geography 102(5), 220-225.

(12)Judson E. (2011) The impact of field trips and family involvement on mental models of the desert environment. International Journal of Science Education 33(11), 1455-1472.

(13)Kamarainen A.M., Metcalf S., Grotzer T., Browne A., Mazzuca D., Tutwiler M.S., Dede C. (2013) EcoMOBILE: Integrating augmented reality and probeware with environmental education field trips. Computers \& Education 68, 545-556.

(14)Kean W.F., Enochs L.G. (2001) Urban field geology for K-8 teachers. Journal of Geoscience Education 49(4), 358363.

(15)Knapp D., Barrie E. (2001) Content evaluation of an environmental science field trip. Journal of Science Education and Technology 10(4), 351-357.

(16)Lebo N., Eames C. (2015) Cultivating Attitudes and Trellising Learning: A Permaculture Approach to Science and Sustainability Education. Australian Journal of Environmental Education 31(1), 46-59.

(17)Levine M., Serio N., Radaram B., Chaudhuri S., Talbert W. (2015) Addressing the STEM gender gap by designing and implementing an educational outreach chemistry camp for middle school girls. Journal of Chemical Education 92(10), 1639-1644.

(18)Lima A., Vasconcelos C., Félix N., Barros J., Mendonça A. (2010) Field trip activity in an ancient gold mine: Scientific literacy in informal education. Public Understanding of Science 19(3), 322-334.

(19)Malbrecht B.J., Campbell M.G., Chen Y.S., Zheng S.L. (2016) Teaching Outside the Classroom: Field Trips in Crystallography Education for Chemistry Students. Journal of Chemical Education 93(9), 1671-1675.

(20)Morag O., Tal R.T. (2012) Assessing learning in the outdoors with the field trip in natural environments (FiNE) framework. International Journal of Science Education 34(5), 745-777.

(21)Nadelson L.S., Jordan J.R. (2012) Student attitudes toward and recall of outside day: An environmental science field trip. Journal of Educational Research 105(3), 220-231.

(22)Patrick P., Mathews C., Tunnicliffe S.D. (2013) Using a field trip inventory to determine if listening to elementary school students' conversations, while on a zoo field trip, enhances preservice teachers' 
abilities to plan zoo field trips. International Journal of Science Education 35(15), 2645-2669.

(23)Peterman K.E. (2008) Field trips put chemistry in context for non-science majors. Journal of Chemical Education 85(5), 645.

(24)Puhek M., Perše M., Šorgo A. (2012) Comparison between a real field trip and a virtual field trip in a nature preserve: knowledge gained in Biology and Ecology. Journal of Baltic Science Education 11(2), 164-174.

(25)Robertson A. (2007) Development of shared vision: Lessons from a science education community collaborative. Journal of Research in Science Teaching 44(5), 681-705.

(26)Stevens S., Andrade R., Page M. (2016) Motivating Young Native American Students to Pursue STEM Learning Through a Culturally Relevant Science Program. Journal of Science Education and Technology 25(6), 947-960.

(27)Sturm H., Bogner F.X. (2010) Learning at workstations in two different environments: A museum and a classroom. Studies in Educational Evaluation 36(1), 14-19.

(28)Tal R.T., Bamberger Y., Morag O. (2005) Guided school visits to natural history museums in Israel: Teachers' roles. Science Education 89(6), 920-935.

(29)Tal R.T., Lavie Alon N., Morag O. (2014). Exemplary practices in field trips to natural environments. Journal of Research in Science Teaching 51(4), 430-461.

(30)Tal R.T., Morag O. (2007) School visits to natural history museums: Teaching or enriching? Journal of Research in Science Teaching 44(5), 747-769.

(31)Tal R.T., Morag O. (2013) A longitudinal study of environmental and outdoor education: A cultural change. Journal of Research in Science Teaching 50(9), 1019-1046.

(32)Townsend V., Urbanic J. (2013) Industrial field trips: An integrated pedagogical framework of theory and practice. International Journal of Engineering Education 29(5), 1155-1165.

(33)Tretinjak C.A., Riggs E.M. (2008) Enhancement of geology content knowledge through field-based instruction for pre-service elementary teachers. Journal of Geoscience Education 56(5), 422-433.

(34)Whitesell E.R. (2016) A day at the museum: The impact of field trips on middle school science achievement. Journal of Research in Science Teaching 53(7), 1036-1054.

(35)Wünschmann S., Wüst-Ackermann P., Randler C., Vollmer C., Itzek-Greulich H. (2017) Learning Achievement and Motivation in an Out-of-School Setting-Visiting Amphibians and Reptiles in a Zoo Is More Effective than a Lesson at School. Research in Science Education 47(3), 497-518.

(36)Zamalloa T., Sanz J., Maguregi G., Fernández M.D., Echevarría I. (2014) Acercar la geodiversidad a través de las salidas de campo en la ESO. Una investigación con el profesorado de ciencias de Bizkaia. Enseñanz̧a de las ciencias 32(3), 443-467.

(37)Zoldosova K., Prokop P. (2006) Education in the field influences children's ideas and interest toward science. Journal of Science Education and Technology 15(3-4), 304-313. 